\title{
TORONTO facial tetrahedron 3D analysis: efficient cephalometric application for surgical mandibular asymmetry, genioplasty, and predictive response to functional dentofacial orthopedic appliances
}

\author{
John Voudouris ${ }^{*}$, Sunjay Suri ${ }^{2}$, Bryan Tompson ${ }^{3}$, James Poulos ${ }^{4}$ and Christos Schismenos ${ }^{5}$ \\ ${ }^{1}$ Clinical Researcher and Associate in Orthodontics, University of Toronto, Discipline of Orthodontics, Toronto, Canada \\ ${ }^{2}$ Chairman and Director, University of Toronto, Faculty of Dentistry, Discipline of Orthodontics, Toronto, Canada \\ ${ }^{3}$ University of Toronto, Faculty of Dentistry, Discipline of Orthodontics, and Director Craniofacial Clinic, Hospital for Sick Children, Toronto, Canada \\ ${ }^{4}$ College of Dentistry, Ohio State University in Columbus, Ohio, USA \\ ${ }^{5}$ College of Dentistry, Department of Orthodontics, Private practice, Agrinio, Greece
}

Simple, accurate, and highly efficient are the words and factors a contemporary orthodontic practice looks for in a cephalometric analysis today. A four-sided Tetrahedron is presented for the orthodontic team to trace easily and allow an active orthodontic clinician to assess, at a glance, a patient's facial pattern and condition. The tetrahedron is suggested as an advancement to the prior Tweed triangle [1]. The reason is a tetrahedron is more representative of the four-sides of the maxilla and mandible. It uses the position of the upper incisor with upper lip, and lower incisor with soft tissue chin as references, simultaneously similar as to how a clinician would apply them chairside.

One problem with dentofacial orthopedic treatment is that fixed functional appliances are often placed indiscriminately in patients with Class II skeletal dysplasia and at all ages. However, a set of cephalometric guidelines or directions, is indicated for proper patient selection to prevent failures [2]. Namely the tetrahedron adds the simple key measurement of CoGnMe shown to provide greater predictability toward more successful orthodontic outcomes with specific types of orthodontic appliances. This not only includes fixed and removable functional appliances, but additionally headgear, and Class II elastics used in skeletal Class II treatment.

A second related problem in the orthodontic profession is a large proportion of orthodontic clinicians (approximately 60\%) report growingly [3] they do not trace cephalometric radiographs for diagnosis and treatment planning $[4,5]$. This is often because it is too time-consuming or complex for the orthodontic team, or they feel it is unnecessary. However, this causes several types of diagnostic, prognostic, and other possible medicolegal complications in dentofacial orthopedic treatment. The purpose of the tetrahedron in the tracing is to be, quick to draw, user-friendly and accurate for the orthodontic staff in order to preserve and attempt to revive the high value of cephalometric measurements under attack, and declining in the orthodontic specialty (Figure 1).

\section{Tetrahedron four walls}

The method used in the Tetrahedron analysis to evaluate the important soft tissue chin position must be, once again, similar to a clinician's technique at chairside, using the natural head position (NHP) at the patient's first consultation appointment. In fact, NHP has been shown to be reproducible long-term [6-11]. By having the patient stand, or sit upright in the orthodontic chair most clinicians draw mentally a perpendicular line to NHP horizontal, through soft tissue subnasale to evaluate the nasolabial angle and soft tissue pogonion. Similarly, the position of the important soft tissue upper lip is evaluated using the same vertical line. Since dentofacial orthopedics combines soft and hard tissue evaluations, the Tetrahedron analysis includes distinctly

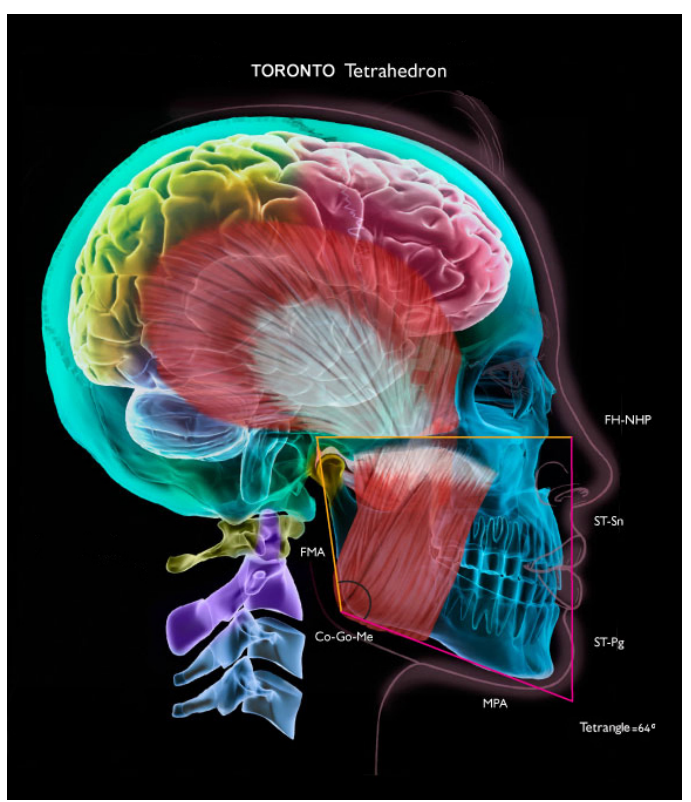

Figure 1. TORONTO Tetrahedron

Correspondence to: John Voudouris, Clinical Researcher and Associate in Orthodontics, University of Toronto, Discipline of Orthodontics, Toronto, Canada; E-mail: jvoud@pathcom.com

Received: April 04, 2017; Accepted: April 18, 2017; Published: April 21, 2017 
Voudouris J (2017) TORONTO facial tetrahedron 3D analysis: efficient cephalometric application for surgical mandibular asymmetry, genioplasty, and predictive response to functional dentofacial orthopedic appliances

one major soft tissue perpendicular vertical wall in conjunction with 3 important hard tissue walls. This is distinctly different compared to the 3 hard tissue lines used in the Tweed triangle.

\section{Tetrahedron wall construction}

Wall One: Corrected Frankfort Plane (or Natural Head Position through Orbitale) Draw the Frankfort plane (FP) ${ }^{\star}$ from the superior point of porion (Po) to orbitale (Or). If the cephalogram is taken with the chin tipped up or down, orient the actual cephalometric radiograph with the Frankfort Plane horizontal (FP) to the bottom of the floor or desk (Figure 1).

Wall Two: Frankfort Perpendicular is drawn through Soft Tissue Subnasale

Draw a perpendicular line starting from Frankfort plane horizontal (or NHPH) through soft tissue subnasale (ST-Sn) extending downward mildly past the lower border of the mandible.

Wall Three: Mandibular Plane to Subnasale Perpendicular

Draw the best-fit line for the lower border of the mandible to represent mandibular plane and connect it with vertical Wall Two.

Wall Four: Draw a line from the superior aspect of the condyle (Condylion, Co) to the corner of the mandible called the gonial angle (Go) following approximately along the line of the ramus. This line is then joined with the mandibular plane line to complete the Tetrahedron.

The ideal alternative to the corrected $\mathrm{FP}^{\star}$ is to use the patient's extraoral facial profile photoimage taken in the natural head position as a template described below to orient the overlayed lateral cephalometric radiograph. NHPH is then drawn through orbitale.

A new Tetrahedron angle is formed at the lower right corner of the tetrahedron, where ethnic differences must also be taken into account. In a caucasian norm the FMA can also be measured more simply by drawing a light supplemental line parallel to the FP horizontal that is then dropped downward to intersect with the gonial angle corner of the tetrahedron (this line continues to also be $90^{\circ}$ to the anterior vertical line of Wall One (Figures 2A, 2B). Mathematically, with a FMA of $26^{\circ}$ the complementary average Tetrahedron angle is $64^{\circ}$. Interestingly, this ideal Tetrahedron angle is identical to the Tweed ideal FMIA located at the upper right corner of the inverted triangle. By having the Tetrahedron angle at the more appropriately located lower right corner of the tetrahedron that is acute $<64^{\circ}$ the clinician obtains a good representation of whether the patient has a long facial pattern and is not likely a good candidate for Class II functional appliance treatment (Figure 2A). The reason is the vertical dimension can be increased with treatment causing the mandible to grow further downward and backward. The Tetrahedron analysis also shows, at a glance, if there is an obtuse Tetrahedron angle $>64^{\circ}$ (Figure $2 \mathrm{~B}$ ) representing a short facial pattern. This makes the patient a good candidate for functional appliances treatment. A comparison of the two tetrahedrons is shown in Figure 2C. Rather than the FMA being inversely proportional generally to muscle activity of the patient, the lower the Tetrahedron angle is more directly associated with lower muscle activity. The higher the Tetrahedron angle is generally, the higher is the muscle activity.

An ideal way to remember the Tetrahedron angle located at the lower right of the tetrahedron is its overall shape. The Tetrahedron angle corresponds to either a narrow facial pattern, or more square or rectangular facial pattern. The Tetrahedron angle in a long facial pattern has a narrow, more acute shape and the tetrahedron appears characteristically elongated in the vertical dimension (approaching

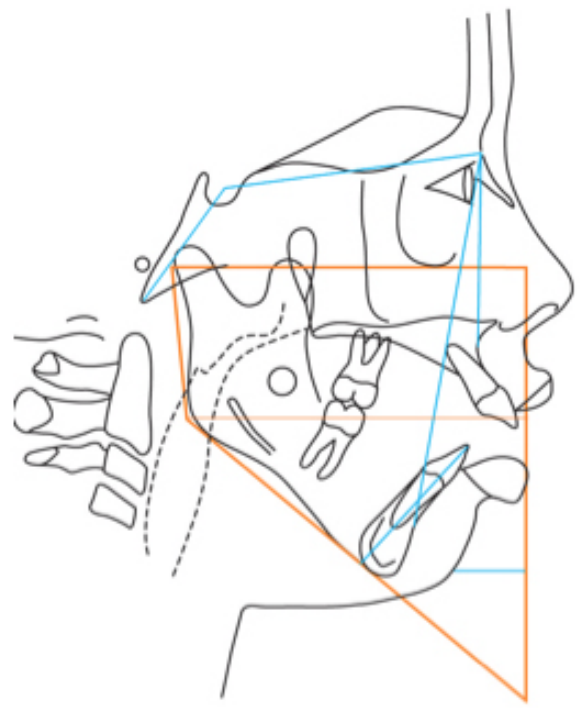

High FMA angle,

low muscle activity, low Tetrahedron angle $=50^{\circ}$

Figure 2A. A male patient of age 12 years presents with a history of CNS and neuromuscular hypoactivity associated with nasopharyngeal obstruction resulting in mouthbreathing, and sleep apnea. Skeletal mandibular retrognathism is associated with a severe Class II division I open bite malocclusion in conjunction with secondary, anterior tongue positioning and posterior molar eruption. The tetrahedron demonstrates a long, narrow face with an acute Tetrahedron angle of $50^{\circ}$ located at the bottom right corner.

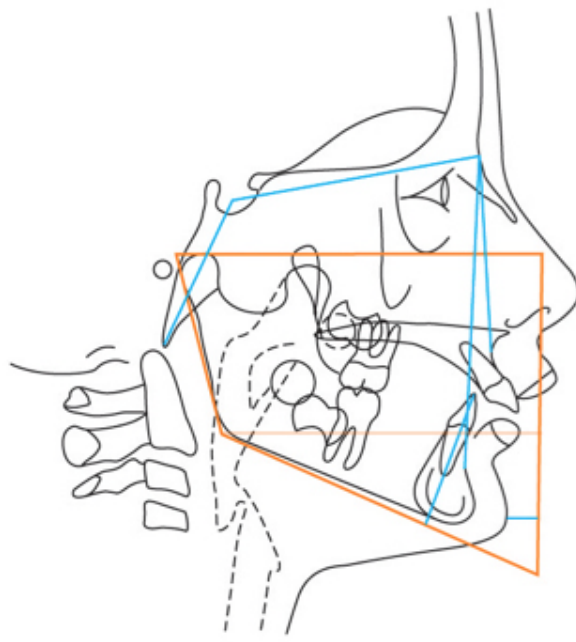

Low FMA angle,

high muscle activity, high Tetrahedron angle $=70^{\circ}$

Figure 2B. In contrast, a female patient of age 12 years presents with a history of CNS and muscle hyperactivity associated with skeletal mandibular retrognathism. A Class II division 1 deep overbite malocclusion is present with a short anterior lower face height due to skeletal restriction of buccal segment eruption associated with a wider more rectangularshaped tetrahedron with an obtuse Tetrahedron angle of 70 degrees. The Tetrahedron angle also has agenerally, a directly proportional association with muscle activity.

a long downward point). The latter, is additionally associated with a short ramus height (Figures $2 \mathrm{~A}, 2 \mathrm{C}$ ). In contrast, the Tetrahedron angle in a short facial pattern has a wider, more obtuse Tetrahedron angle where the tetrahedron shape is skewed toward a rectangular-shape horizontally. The acute Tetrahedron angle overall, demonstrates the dolicofacial "eagle-like" appearance while the more obtuse Tetrahedron angle represents the brachyfacial or more "rectangular-face" appearance. 


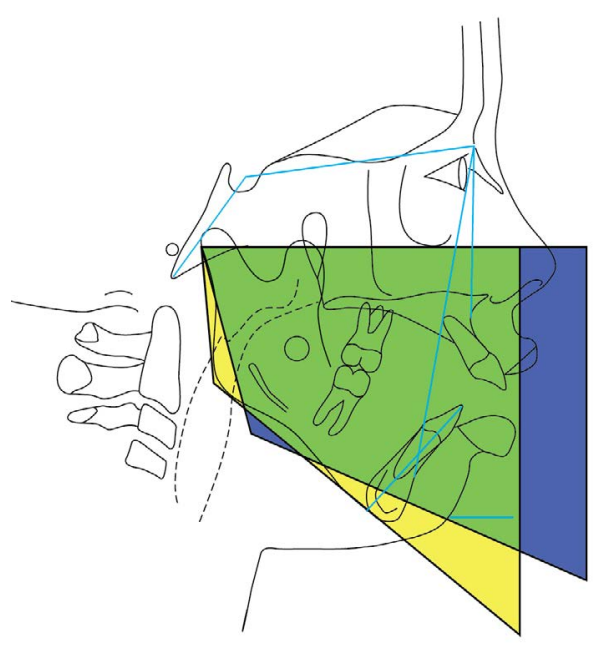

Figure2C. Superimposition: Tetrahedron Angle $50^{\circ}$ in Yellow and $70^{\circ}$ overlay in Blue The distinct differences in the heights and depths of the two Tetrahedrons is shown between a high angle facial pattern in yellow versus a low angle facial pattern in blue (FPor NHPH superimposition).

The ideal soft tissue upper lip position is $3 \mathrm{~mm}$ anterior to the vertical line perpendicular to the FP (or NHPH) and the upper incisor to NA is $3 \mathrm{~mm}$. To evaluate chin position in a particular manner to match actual chairside evaluation, a short horizontal perpendicular is drawn from the vertical line of Wall One to the soft tissue pogonion (ST-Pg) in the Tetrahedron analysis. The ideal soft tissue chin position is 0 to $-2 \mathrm{~mm}$ posterior to the vertical FP perpendicular (or NHPH) used in conjunction with an IMPA of $90^{\circ}+3^{\circ}$. In this manner the tetrahedron analysis uses both the upper and lower jaws as references in conjunction with the upper and lower incisors. It is important that all other distances and angles used regularly by an individual clinician can continue to be applied as adjunctive measurements to the Tetrahedron Analysis. (see below)

\section{Tetrahedron advancement: Building upon the tweed triangle principles}

The Tetrahedron Analysis is part of a face and smile planning discipline that includes a new EMS Angle classification (taking into consideration Etiology, Muscles-viscoelastic soft tissues, and Skeletal factors). The Tetrahedron is shown as a suggested advancement overlay to the Tweed Triangle (Figure 3).

The Tweed philosophy developed by Charles H. Tweed, as part of the highly respected Tweed Foundation in Tucson, Arizona includes one of the most eloquent and original cephalometric analyses in orthodontics. The Tweed Triangle gives particular attention to the angle of the face, as low or high angle, and relates it to the critical lower incisor position [1]. This is central for diagnosis, treatment planning, long-term alignment maintenance and stability. In addition, a highly disciplined Tweed biomechanics technique course is part of the Tweed philosophy. Further Tweed foundation advancements include the Total Space Analysis, Read-out and many others with contributions by outstanding leaders in orthodontic education, over many decades, continuing today.

The Tweed Triangle is composed of the angles FMA, IMPA and FMIA. With a caucasian FMA average of $26^{\circ}$ and upright lower incisors at $90^{\circ}$ the ideal FMIA angle is $64^{\circ}$ ideally. This is important not only in extraction, but also for non-extraction, and dentofacial orthopedic treatment with functional appliances. The general application of the excellent principles and concepts of the Tweed philosophy are the reasons they continue to resonate worldwide.

The Tweed Triangle cephalometrically was particularly ingenious because at minimum, it was simple, relational and it was diagrammatic. The Triangle formed by the hard tissues of the face made it highly visual in two dimensions, rather than simply a line measurement of distance, or one angle alone. A Tweed orthodontist having the drawing of the Tweed Triangle on a cephalometric tracing, with a preliminary glance, has at a minimum a first appreciation of both the patient's skeletal and dental condition. This specifically includes Frankfort Plane to mandibular plane angle, and the lower incisor to the mandibular plane angle respectively. The Tweed Triangle's simplicity and efficiency is important and the Tetrahedron analysis applies the same objectives.

One of the main difficulties with Class II functional appliance treatment has been the ability to select patients accurately to assure greater treatment success. In the diagnosis of skeletal Class II retrognathia found commonly with a deep overbite treated with fixed or removable functional orthopedic appliances, the Tweed Triangle is highly applicable. The selection of patients with an FMA angle ideally less than or equal to 26 degrees for example is critical during functional mandibular advancement. These patients are considered "good growers" for functional appliance treatment. The FMA is important since differential dental eruption of the mandibular buccal segments is encouraged with functional appliances. This generally, increases the vertical dimension and FMA. In addition, the lower incisor position should have ideally an IMPA less than or equal to $90^{\circ}+3^{\circ}$ since there is a tendency toward lower incisor proclination with functional orthopedic appliances.

For patients with an IMPA at the higher range of $93^{\circ}$ it is recommended that negative lower incisor bracket torque of $-5^{\circ}$ in conjunction with interproximal reduction and elastic chain for incisor retraction be preformed prior to or during functional appliance treatment. Further, to address the transverse plane, maxillary expansion with pre-functional RME is additionally recommended to prepare to house and maintain the wider portion of the mandibular dental arch that is functionally advanced.

\section{FP perpendicular and CoGnMe angle: Two additions to the tweed triangle}

The two main changes suggested in the Tetrahedron analysis are located anteriorly and posteriorly to better represent the middle and lower face. The first is essentially an additional soft tissue vertical line

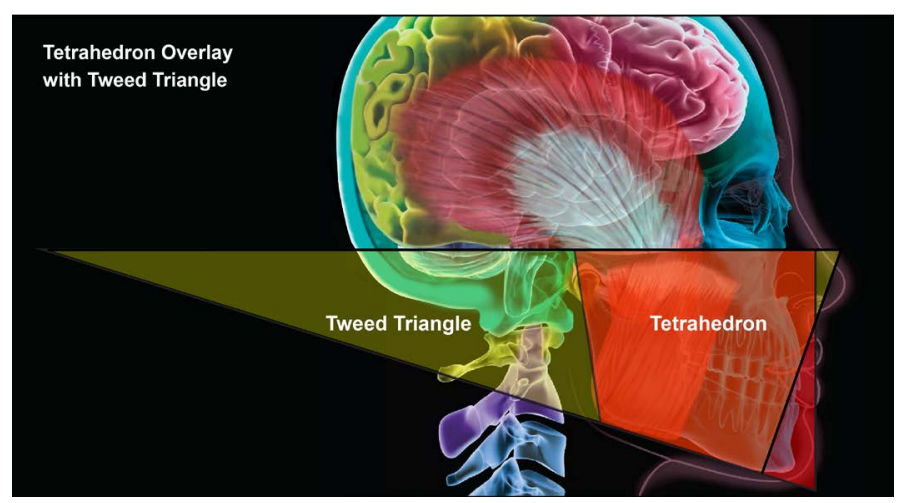

Figure 3. Tetrahedron analysis (red) superimposed over the Tweed Triangle (yellow) demonstrates the four-dimensions compared to three-dimensions respectively. 
Voudouris J (2017) TORONTO facial tetrahedron 3D analysis: efficient cephalometric application for surgical mandibular asymmetry, genioplasty, and predictive response to functional dentofacial orthopedic appliances

to begin to form a tetrahedron. It is used to determine practically the important soft tissue upper lip position and (ST-ULp), and severity of mandibular retrognathia (ST-Pg) anteroposteriorly. This is especially useful for diagnosis and treatment planning in functional appliance or headgear treatment such as the amount of mandibular advancement needed with the functional appliance. For example, it determines whether the functional mandibular advancement should be completed in one stage, or progressively in two or more stages to prevent excessive strain to the TMJ in patients with severe retrognathia and overjet. Two millimetre advancement C-stops can be placed onto the fixed functional appliance for example to provide progressive, continuous advancement of the mandible. Soft tissue chin evaluation was the primary rational for using the Frankfort Plane perpendicular passing through soft tissue subnasale.

The second advancement located at the posterior region relates more recently to the condylion-gonion-mandibular plane angle, CoGnMe. In a study by Baccetti and Franchi (2006) it was demonstrated that the CoGnMe angle is a key single measurement toward predicting treatment outcomes [2]. The reason is the CoGnMe angle has a good statistical correlation, for selecting patients that are good responders to functional orthopedic treatment. The suggested ideal CoGnMe angle is $<126^{\circ}$. In addition, it can be combined in conjunction with the FMA and other measurements (lower face height etc.) to reasonably select patients for functional orthopedic appliance treatment. This is the primary rationale for applying CoGnMe to the Tetrahedron cephalometric analysis. Other diagnostic indicators for selecting and timing functional appliance treatment are: 1) patient age, 2) cervical vertebrae maturation (CVM), 3) height, and 4) carpal development.

The second reason for using the Co-Go line is to form and close the walls of the tetrahedron. This better describes diagrammatically the maxilla and mandible in a simple 4-sided geometric form. Both the Tetrahedron and Tweed analyses use a similar Frankfort plane, however, by adding the important posterior aspect of the mandible, the middle and lower face are represented more completely by the 4 -sided tetrahedron compared to a three-sided, inverted triangle. It is important to be aware that the mandibular plane and ramal plane essentially using condylion are relatively stable. However, it is recognized that bone formation locally at gonial angle has the potential for turnover with significant condylar trauma, and to be highly modified since it is the insertion site of the active masseter muscle. With functional appliances treatment generally increasing the vertical dimension and reducing masseter muscle activity [12], this may modify the gonial angle and also affect the antegonial notch region. However, any changes in the gonial angle are highly localized and small compared to the more identifiable mandibular plane and ramus plane.

The alternative use of NHPH was a concept applied by Moorrees at Harvard university and several subsequent investigations have found it to be highly reproducible [13]. The extraoral profile photoimage taken in the NHP can be useful in drawing the NHPH through orbitale. The profile photoimage is taken with the NHP by using a mirror in front of the patient asking the patient to look directly at their eyes in the reflection. An alternative is to look out naturally toward the horizon. Another method is to use the patient's posture to achieve the NHP by asking the patient to walk forward 3-steps and back 3-steps to establish head posture prior to taking the profile photoimage. It is notable that the Frankfort Plane on the cephalogram often approximates the $\mathrm{NHPH}$. Indeed, the Frankfort plane is more easily attained since it is can at times be difficult to obtain the NHP in a cephalostat.

\section{Adjunctive measurements used in the tetrahedron}

Although the Tetrahedron analysis was developed to be overall simple and easy to use, many other customized measurements may be added as supplements. The adjunctive analyses used with the Tetrahedron analysis include the evaluation of upper incisor torque, upper incisor to palatal plane for upper incisor angle, interincisal angle, along with the nasolabial angle, for face planning and dental display. The upper central incisors, used as a key reference, are exposed ideally $3 \mathrm{~mm}$ in lip repose [14]. Other important measurements are applied including the Harvold analysis of maxillary and mandibular unit length differences (average 22 $\mathrm{mm}$ ) used in the McNamara analysis, the measurement of anterior lower face height (aLFH average $=64+4 \mathrm{~mm}$ ), $\mathrm{UFH}: \mathrm{LFH}$ ratio $=45: 55$, in conjunction with the Steiner analysis for SNA and SNB to determine the ANB angle. The Tetrahedron cephalometric analysis may also be supplemented by other measurements including horizontal distance for the lower incisor to NB line, lower incisor to A-Pg line, occlusal plane angle, and the WITS analysis.

Although the CoGoMe was suggested by Baccetti and Franchi (2006) as the key single measurement for prediction of orthopedic outcome the Tetrahedron analysis relies on combining this specifically with FMA, aLFH, UFH:LFH ratio, cranial base angle, SNB, SNA ANB, Harvold unit length difference and others.

\section{TORONTO facial tetrahedron 3D wedge}

With the advent of 3D imaging such as cone-beam CT (CBCT) scanning, cephalometric analyses have become three-dimensional. The mandibular symphyseal angle [15] can also be measured from a submental vertical radiograph and can be then added to the 2 tetrahedrons on the right and left sides of the face. The Frankfort plane and the ramal plane are then also placed to complete the formation of a 3D Tetrahedron wedge (Figure 4). Patients with a long, anterior lower face height are expected to have a more acute and narrow symphyseal angle than a patient with a short, anterior lower face height. The long facial pattern will additionally demonstrate from a three-quarter perspective a vertically pulled or elongated facial $3 \mathrm{D}$ wedge while the short facial pattern will demonstrate a more vertically compressed or squared-off type of $3 \mathrm{D}$ wedge.

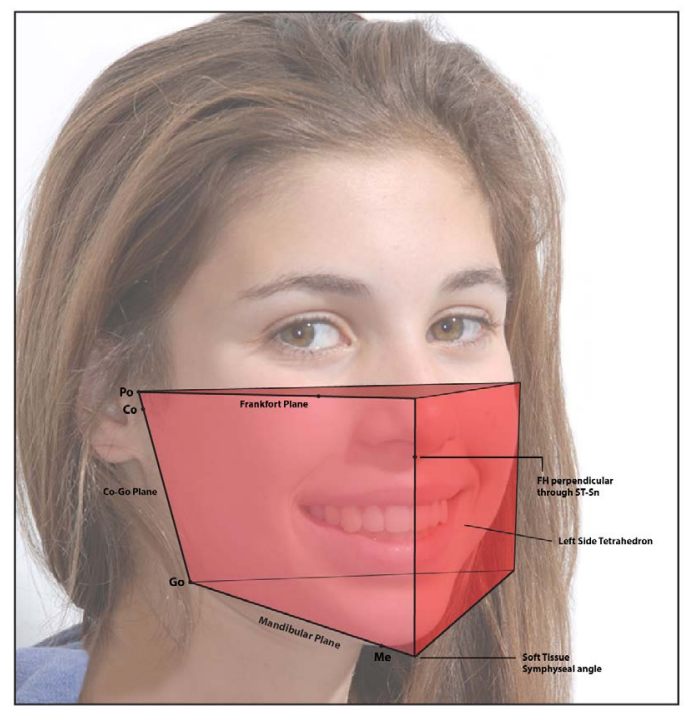

Figure 4. Facial Tetrahedron Wedge in 3D. A wedge is formed using the symphysea angle, the two tetrahedrons on the right and left sides of the face applying the ramal plane, and the Frankfort plane corrected horizontally. This 3D facial analysis can be applied in evaluating CBCT scans 
Voudouris J (2017) TORONTO facial tetrahedron 3D analysis: efficient cephalometric application for surgical mandibular asymmetry, genioplasty, and predictive response to functional dentofacial orthopedic appliances

\section{Clinical applications of the TORONTO facial tetrahedron 3D analysis}

The Symphyseal Angle is highly useful in calculating the amount of mandibular asymmetry and thereby the precise amount of surgical correction required on each side of the mandible. This angle is used to calculate the amount of rotation required for Corrected oblique radiographs to determine the difference in size between the right side of the mandible and the left side of the mandible for surgery.

A more obtuse Symphyseal Angle is indicated for advancement genioplasty since it would provide a greater blood supply to the advanced chin button for healing.

Since a more acute Co-Gn-Me angle $<128^{\circ}$ has been correlated with a Good Response to Functional Dentofacial Orthopedic treatment, and the hypothesis is that the Co-Gn-Me angle negatively correlated with the Symphyseal angle, then Good Responders to Functional appliance treatment would be negatively correlated with the Symphyseal Angle. The Symphyseal angle provides another guidline in predicting diagnostically the response to functional appliance treatment prior to initiating the treatment.

\section{Summary}

A relatively simple-to-draw, four-sided geometry is recommended to represent a more realistic and practical facial form for efficient cephalometric evaluation in a busy orthodontic practice. This analysis uses the Frankfort plane (FP), FP perpendicular line anteriorly and the CoGoMn posteriorly. The ideal average angle for caucasians formed at the lower right corner of the tetrahedron is $64^{\circ}$ (intersection of the FP perpendicular line and mandibular plane) in the Tetrahedron analysis. This coincidentally matches with the ideal FMIA located in the upper right corner of the inverted Tweed Triangle. A Tetrahedron Wedge is additionally recommended for comprehensive, $3 \mathrm{D}$ cephalometric analysis of CBCT scans. This 3D Tetrahedron analysis has significant applications in Asymmetry Surgery with genioplasty and in diagnostically predicting response to functional appliance treatment prior to initiating such treatment.

\section{References}

1. Tweed $\mathrm{CH}$ (1969) The diagnostic facial triangle in the control of treatment objectives. Am J Orthod 55: 651-657. [Crossref]

2. Franchi L, Baccetti T (2006) Prediction of individual mandibular changes induced by functional jaw orthopedics followed by fixed appliances in class II patients. Angle Orthod 76(6): 950-954. [Crossref]

3. Gottlieb EL, Nelson AH, Vogels DS 3rd (1997) 1997 JCO Orthodontic Practice Study Part 1. Trends. J Clin Orthod 31: 675-684. [Crossref]

4. Keim RG, Gottlieb EL, Nelson AH, Vogels DS 3rd (2005) 2005 JCO Orthodontic Practice Study. Part 1: trends. J Clin Orthod 39: 641-650. [Crossref]

5. Keim RG, Gottlieb EL, Nelson AH, Vogels DS 3rd (2013) 2013 JCO Orthodontic Practice Study. Part 1: trends. J Clin Orthod 47: 661-680. [Crossref]

6. Cooke MS, Wei SH (1988) The reproducibility of natural head posture: a methodological study. Am J Orthod Dentofacial Orthop 93: 280-288. [Crossref]

7. Cooke MS (1990) Five-year reproducibility of natural head posture: a longitudinal study. Am J Orthod Dentofacial Orthop 97: 489-494. [Crossref]

8. Chiu CS, Clark RK (1991) Reproducibility of natural head position. J Dent 19: 130131. [Crossref]

9. Peng L, Cooke MS (1999) Fifteen-year reproducibility of natural head posture: A longitudinal study. Am J Orthod Dentofacial Orthop 116: 82-85. [Crossref]

10. Bister D, Edler RJ, Tom BD, Prevost AT (2002) Natural head posture--considerations of reproducibility. Eur J Orthod 24: 457-470. [Crossref]

11. Pereira AL, De-Marchi LM, Scheibel PC, Ramos AL (2010) Reproducibility of natura head position in profile photographs of children aged 8 to 12 years with and without the aid of a cephalostat. Dental Press J Orthod 15.

12. Voudouris JC, Woodside DG, Altuna G, Angelopoulos G, Bourque PJ, et al. (2003) Condyle-fossa modifications and muscle interactions during Herbst treatment, Part 2. Results and conclusions. Am J Orthod Dentofac Orthop 124: 13-29.

13. Moorrees CF, Efstratiadis SS, Kent RL Jr (1991) The mesh diagram for analysis of facial growth. Proc Finn Dent Soc 87: 33-41. [Crossref]

14. Gianelly AA (2000) Bidimensional technique theory and practice. GAC International.

15. Weber DW, Fallis DW, Packer MD (2013) Three-dimensional reproducibility of natural head position. Am J Orthod Dentofacial Orthop 143: 738-744. [Crossref]

Copyright: (C2017 Voudouris J. This is an open-access article distributed under the terms of the Creative Commons Attribution License, which permits unrestricted use, distribution, and reproduction in any medium, provided the original author and source are credited. 